\title{
A Survey on Applications of Neural Networks and Genetic Algorithms in Fault Diagnostics for Antenna Arrays
}

\author{
Subhash Mishra ${ }^{1}$, R.N. Yadav ${ }^{2}$, R.P. Singh ${ }^{3}$ \\ ${ }^{1}$ Research Scholar,${ }^{2}$ Professor,${ }^{3}$ Professor \\ Department of Electronics and Communications Engineering \\ Maulana Azad National Institute of Technology, Bhopal, INDIA
}

\begin{abstract}
Fault diagnosis in antenna arrays implies locating the faulty or defective elements in the array. Smart antenna arrays use digital beamforming and allow for array failure corrections. The defective elements of such antenna arrays therefore need not be replaced unlike the traditional analog approach. The smart antennas can thus support cost-effective solutions instead of replacing the hardware. The traditional analytical methods find it tedious to handle fault finding problems related with antenna arrays. Neural networks are nonlinear in nature. They can map the non linear behavior of smart antennas arrays and perform fault diagnosis with considerable time reduction. Genetic algorithms have also been applied very successfully in locating faults in the antenna array. In this review, the applications of neural networks and genetic algorithms in fault diagnosis of antenna arrays are summarized.
\end{abstract}

Keywords: Adaptive array; Smart Antenna; Fault Diagnosis; Neural Network; Genetic Algorithm.

\section{Introduction}

The smart antenna technology has evolved as a powerful technique enabling solutions to some of the most significant issues related to the modern day wireless communications and therefore has become a favourite subject of study amongst the researchers. Smart antennas utilize spatial diversity aspects which is effective in reduction of co-channel interference and fading. Smart antennas use antenna arrays with signal processing algorithms for identifying the signals spatially [1][2][3]. The smart antenna techniques are expected to have a major impact on the efficient use of the spectrum and the minimization of the cost of deploying future wireless networks [4]. Multiple antennas can be used to form beams which cover the whole cell site. This method is known as cell sectoring. Switched beam antenna arrays are extension of cell sectoring. The adaptive antenna arrays however are able to steer multiple beams thus extending the coverage range. They are able to improve the channel capacity and spectrum efficiency of mobile communications systems [5][6][7].Smart antenna arrays can locate each user and can cover groups of users. The ability to steer beams toward users and point nulls towards other mobiles helps to reduce interference [5][7].Detailed descriptions on various aspects related with the concepts and applications of antennas and antenna arrays can be found in [1-3][5][7].

Antenna arrays are used in several applications which include wireless communications, radar, sonar and satellite communications. These antennas have several radiating elements sometimes in hundreds. The possibility of failure in some of them increases due to the large number of elements. Faults in arrays disturb the radiation pattern [20][21][26][31] and also affect the input impedance [23].Traditional analytical methods used earlier for locating the faults in antenna arrays are tedious and time consuming. Information on the number and location of the faulty elements is required and these methods generally find it difficult to handle fault diagnosis. Hence new approaches are required [20-22][25][26].Built in monitoring and calibration systems are complex and costly[33].The digital beamforming in smart antenna arrays provides possibility of failure correction and thus a cost-effective solution to hardware replacement [20].Artificial neural networks (ANNs)[10][14] are non linear and use simple mathematical operators. They are able to locate faults by performing mapping between the damaged radiation pattern/s with the position of the fault element/s. ANNs are flexible and robust enough to identify the faults in arrays. By using ANNs it is possible to recalculate and produce the radiation pattern close to the original pattern [24][25][26][29][31-32]. The adaline [24], multilayer perceptron (MLP) [21][2526][33]the radial basis function (RBF) neural networks [31][32] and the discrete mean field neural network based on Vidyasagar net [22] and have been found quite effective in finding faults in the antenna arrays. Genetic algorithms have also been very effectively used in fault diagnosis of arrays as mentioned ahead [20][21][23][30]. A combination suggested by authors in [29] utilizes features of both the approaches; the neural network and the genetic algorithm. Section II describes the mathematical nature of the fault finding problem. Section III presents the configurations of the multi layer perceptron (MLP) and the radial basis function (RBF) neural networks. Section IV includes basics principle of genetic algorithm. Various fault finding applications related to neural networks and genetic algorithm are covered in section V. Section VI concludes the paper. 


\section{Fault Finding}

Faults in antennas distort the radiation pattern and cause sharp variations in the field intensity around an antenna array which increases the side lobes and ripple level of the power pattern [26][29]. The patterns can be restored however through readjustment of the excitations of the non-defective elements to produce a pattern with respect to the original [21][30]. Analytical approaches can perform the compensation by numerically finding a new set of excitations of the functional elements. These methods require the information on the number and the location of the failed elements in the array [21]. Since a failed array can be considered as a nonuniformly spaced array, analytical approaches generally find it difficult to deal with this kind of problem. Numerical algorithms also have been used to correct the deformed patterns. However, the geometrical layout of the remaining functional array elements and of the desired beam shape presents difficulty [20].Measurements of the initial radiation pattern and the that of a small number of spatial directions of the damaged radiation pattern (from base station) can be helpful in fault diagnosis [21].The beamforming approach using both amplitude and phase is suitable for the redistribution of weights for correcting the damaged pattern [20][24]. The expression for array factor (AF) for a linear antenna array with $N$ elements as given in [20] [24] is,

$$
\begin{aligned}
& A F=\omega^{T} S\left(\theta, \theta_{m}\right) \\
& \omega=\left\{\omega_{1}, \omega_{2}, \omega_{3}, \omega_{4}, \ldots \ldots \omega_{N}\right\}^{T}
\end{aligned}
$$

Here $\omega$ is the weight vector, $S$ is the steering vector, $\theta$ and $\theta_{m}$ are the direction variable and main beam direction, respectively

$S=\exp \left\{j k d\left(n-\frac{N-1}{2}\right) \cdot\left(\cos \theta-\cos \theta_{m}\right)\right\}$

In the event of the $m^{\text {th }}$ element failure, its weight $\omega_{m}$ is assumed to be zero. Suitable techniques can be applied to enable corrections in the side lobes and the radiation patterns.

\section{Neural Networks}

Artificial neural networks (ANNs) have been applied in a variety of applications related with antenna arrays [15]. The concept and development of artificial neural network is motivated from the concept of biological neurons of human brain [10]. Neural networks provide simple general purpose mathematical tools. Their enormous parallel processing potential and capability to provide fast solutions have made them popular [8]. After training, neural networks can perform cumbersome operations in real time. Details on principle of working, structures and applications of neural networks are described in [8][10][14].Some reviews on neural network applications in digital communications, antenna arrays and smart antennas are available in [13][17][18][19][35]. The non linear neural networks are able to understand the behavior of the antenna arrays. The neural network methodologies for fault diagnostics in antenna arrays are based on the mapping between the damaged radiation pattern/s with the position of the fault element/s. This enables them to detect the location and the number of the faulty elements as shown in [24][25][26][29][31-33]

\section{III.1. Multi Layer Perceptron Neural Networks}

A multilayer perceptron (MLP) is a feed forward, non linear neural network which can have single or multiple hidden layers. The configuration of a multilayer perceptron is shown in figure1. Each neuron of a layer is connected to each neuron of the next layer. This makes forward transmission from the input layer to the

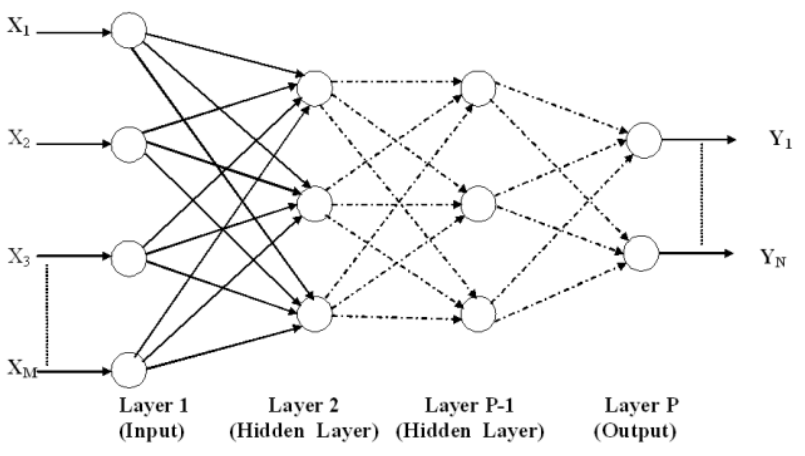

Figure 1. Multi Layer Perceptron Neural Network

output layer through the hidden layers possible [10][14].Each neuron is characterized by an activation function and its bias, and each connection between two neurons by a weight factor. A non linear function like sigmoid or tansigmoid is normally used as the activation function for a hidden layer neuron [10][14].The input signals 
propagate gradually from the input layer through the hidden layers up to the output layer. The error signals that originate at the output can move layer wise backward through the network. The output [14][34] of the $p^{\text {th }}$ layer can be written as follows,

$Y_{p}=F\left(W_{p} Y_{p-1}+B_{p}\right)$

where $Y_{p}$ and $Y_{p-1}$ are outputs of the $p^{\text {th }}$ and $(p-1)^{\text {th }}$ layer respectively, $W_{p}$ is a weight matrix between $(p-1)^{\text {th }}$ and $p^{\text {th }}$ layer and $B_{p}$ is a bias matrix between the $p^{\text {th }}$ and $(p-1){ }^{\text {th }}$ layer. $F$ is the activation function of each neuron. The training algorithms normally associated with MLP are back propagation (BP), LevenbergMarquardt (LM) or quasi-Newton.

\section{III.2. Radial Basis Function Neural Networks}

A radial basis function (RBF) neural network is a non linear, feed forward network which normally has three layers, an input layer, a hidden layer and an output layer. The input layer contains the parameters of the basic function [10][14]. The transformation from the input layer to the hidden layer is non linear using a radial

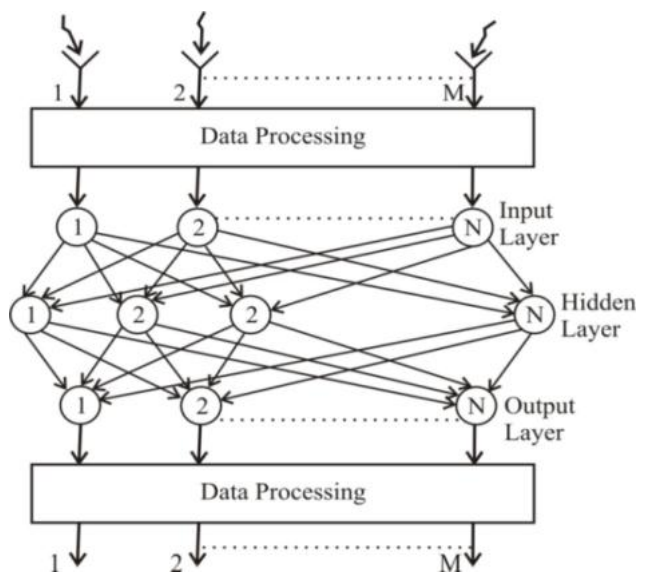

Figure 2. Radial Basis Function Neural Network [16]

basis function while the hidden layer to the output layer is linear [3][10][11] as shown in figure 2 [12][16]. The radial basis functions can be viewed as curve fitting problem in high-dimensioned space. Their ability to produce outputs to previously unseen inputs by interpolating between the unseen inputs in the training phase and their capability for reduction in training set makes them very suitable for the applications in antenna arrays [3][9][10][11][12][16].

\section{Genetic Algorithms}

Genetic algorithms are stochastic optimization algorithms which have very wide applications [20]. They use binary coding and binary genetic operations based on natural evolution and make use of tools that work well in nature. They provide sophisticated search algorithms for complex mathematical search spaces. Thus genetic algorithms are capable of arriving at an optimal solution without the benefit of explicit knowledge about the solution space. Genetic algorithms find applications in antenna array fault diagnostics [20$21][23][27]$. They compare the measured radiation patterns of the array with the configuration of faulty and functional elements. The configuration used in this comparison depends on the chromosome of the genetic algorithm [20].The minimization of this cost function using genetic algorithms allows helps to calculate radiation pattern that is closer to the measured one. This enables to obtain the number and the location of the defective elements. In the case of partial failures also, this chromosome is able to know the grade of failure of the array elements [20-21] [30].Genetic algorithms can also be used with neural networks for locating faults in arrays [29].

\section{Fault Finding in Antenna Arrays Using Neural Networks and Genetic Algorithms}

Authors in [20] have presented an effective method based on the genetic algorithm for array failure correction of arbitrary digital beamforming arrays. A 32- element antenna array based on Dolph-Chebyshev linear antenna design (with SLL of $-35 \mathrm{db}$ ) has been considered in the study. The representation of the array weights has been by vector of complex numbers. The decimal linear crossover is employed. Performance of the three schemes namely adjacent-fitness-paring (AFP), best-mate-worst (BMW) and emperor-selective (EMS) are 
studied. It is seen that the success of correcting a damaged pattern depends on the original weighting the number of failed elements. The method has been found effective for array failure correction of single, double or triple element failure cases. A technique to locate defective elements in planar arrays by using some samples of the degraded far-field power pattern is presented in [21] where authors have considered a planar array $(8 \times 8$ elements) in a rectangular grid with $0.7 \lambda$ inter element spacing. Genetic algorithm has been used to minimize the square of the difference between the far-field power pattern obtained for a given configuration of failed elements and the measured one. The method [21] is effective in complete as well as partial failure cases. It has been found that the probability of finding the right solution increases with the number of samples. Authors in [22] have proposed use of a discrete asynchronous version of the Vidyasagar mean field neural network, to identify the faulty element in a large array and compared the performance of the method with the standard conjugate gradient method. A 25-element antenna array has been considered in the study.

The failure of radiating elements in an antenna array not only degrades its radiation pattern, but also affects its input impedance. In [23] authors have presented a method of element failure detection that does not require pattern phase measurements. Mutual coupling among the elements is into account. Measurements of the pattern amplitude and the input impedance of the antenna are combined. A 12-slot linear array antenna implemented in waveguide operating at $9.375 \mathrm{GHz}$ has been considered here. Success rates of $100 \%$ for single faulty element, $92 \%$ for two faulty elements and $88 \%$ for three faulty elements have been reported. Authors in [24] have suggested an adaptive neuronal system for locating faults in antenna arrays. A 16-element linear antenna array has been considered in the study. The neural method has the advantage of faster convergence. Adaptive linear neurons (Adalines) have been used quite effectively.

Authors in [25] have proposed a method using a three layer multilayer perceptron based artificial neural network (ANN) to identify the fault elements in linear array antennas. The neural network performs the mapping between the radiation pattern of the faulty array and the faulty element position. The response of the neural network after training is very fast in locating completely faulty as well as partially faulty elements. A 5element binomial array has been considered here. The technique has been found very effective and has been suggested by the authors for use in planar arrays as well. Authors in [26] have extended the idea further to present a flexible approach of locating fault elements in antenna arrays using artificial neural networks employing MLPs trained in back propagation.

\section{Table 1 Various Fault Finding Methods Using Neural Networks And Genetic Algorithms}

\begin{tabular}{|c|c|c|c|}
\hline S. No. & Applications & Method & Antenna Array \\
\hline 1 & $\begin{array}{l}\text { Array failure correction with a genetic algorithm } \\
\text { [20] }\end{array}$ & Genetic algorithm & $\begin{array}{l}\text { 32-element linear array, inter } \\
\text { element spacing } d=0.5 \lambda\end{array}$ \\
\hline 2 & $\begin{array}{l}\text { Finding defective elements in planar arrays using } \\
\text { genetic algorithms[21] }\end{array}$ & Genetic algorithm & $8 \times 8$ planar array, $d=0.7 \lambda$ \\
\hline 3 & $\begin{array}{l}\text { Efficient faulty element diagnostics of large } \\
\text { antenna arrays by discrete mean field neural nets } \\
{[22]}\end{array}$ & $\begin{array}{l}\text { Discrete version of } \\
\text { Vidyasagar mean field neural } \\
\text { network }\end{array}$ & 25-element linear array \\
\hline 4 & $\begin{array}{l}\text { Waveguide fed longitudinal slot array antennas: } \\
\text { Fault diagnosis using measurements of input } \\
\text { impedance [23] }\end{array}$ & Genetic algorithm & 12- slot linear array \\
\hline 5 & $\begin{array}{l}\text { Antenna array adjust with adaptive neuronal system } \\
\text { [24] }\end{array}$ & Adaline neural network & 16-element linear array \\
\hline 6 & $\begin{array}{l}\text { Finding failed element positions in linear Antenna } \\
\text { arrays using neural networks [25] }\end{array}$ & $\begin{array}{l}\text { Multi layer perceptron neural } \\
\text { network }\end{array}$ & 5- eleme \\
\hline 7 & $\begin{array}{l}\text { An ANN application for fault finding in Antenna } \\
\text { arrays [26] }\end{array}$ & $\begin{array}{l}\text { Multi layer perceptron neural } \\
\text { network }\end{array}$ & $\begin{array}{l}\text { 16-element linear micro strip } \\
\text { array, } d=0.3 \lambda\end{array}$ \\
\hline 8 & $\begin{array}{l}\text { A comparison among several techniques for finding } \\
\text { defective elements in antenna array [27] }\end{array}$ & $\begin{array}{l}\text { Case based reasoning, Neural } \\
\text { networks, Genetic algorithm }\end{array}$ & $\begin{array}{l}\text { 100-element linear array, } \\
d=0.5 \lambda\end{array}$ \\
\hline 9 & $\begin{array}{l}\text { Element failure detection in linear antenna array } \\
\text { using case based reasoning [28] }\end{array}$ & $\begin{array}{l}\text { Case based reasoning, SOM } \\
\text { neural network }\end{array}$ & $\begin{array}{l}\text { 100-element linear array, } \\
d=0.5 \lambda\end{array}$ \\
\hline 10 & $\begin{array}{l}\text { A genetically trained neural network application for } \\
\text { fault finding in antenna arrays [29] }\end{array}$ & $\begin{array}{l}\text { Multi layer perceptron with } \\
\text { Genetic algorithm }\end{array}$ & $\begin{array}{l}\text { 16- element linear array, } \\
d=0.3 \lambda\end{array}$ \\
\hline 11 & $\begin{array}{l}\text { Rapid method for finding faulty elements in } \\
\text { antenna arrays using far field pattern samples [30] }\end{array}$ & Based on Genetic algorithm & $\begin{array}{l}\text { 100-element linear array, } \\
d=0.5 \lambda\end{array}$ \\
\hline 12 & $\begin{array}{l}\text { Fault diagnosis of planar antenna arrays using } \\
\text { neural networks [31] }\end{array}$ & $\begin{array}{l}\text { Radial basis function and } \\
\text { Probabilistic neural networks }\end{array}$ & $5 \times 5$ and $8 \times 8$ planar arrays \\
\hline 13 & $\begin{array}{l}\text { Using neural networks for fault detection in planar } \\
\text { antenna arrays [32] }\end{array}$ & $\begin{array}{l}\text { Radial basis function and } \\
\text { Probabilistic neural networks }\end{array}$ & $5 \times 5$ and $8 \times 8$ planar arrays \\
\hline 14 & $\begin{array}{l}\text { Element failure diagnosis in a planar antenna array } \\
\text { by the use of neural networks [33] }\end{array}$ & $\begin{array}{l}\text { Multi layer perceptron neural } \\
\text { network }\end{array}$ & $6 \times 6$ planar array, $d=0.9 \lambda$ \\
\hline
\end{tabular}


Artificial neural networks do not require the iterative process of traditional methods. The ANN forms a mapping between the damaged radiation patterns with the positions of the fault element/s in the array. The method is successfully tested for a linear array and the can be used for planar arrays also as suggested in the study. The presented technique can be used in the base stations to detect the number and location of the faulty array elements in space platforms. In [27], authors have compared several techniques used for detecting the locations and the numbers of the faulty elements in arrays. A 100 - element antenna with $0.5 \lambda$ interspacing has been used in the study. The methods such as Woodward-Lawson method, neural networks, genetic algorithm and others are compared. It is seen that the Woodward-Lawson method achieves good results but requires many measurements of both the amplitude and phase of the degraded patterns, and therefore offers limited applicability. The genetic algorithm technique has been very effective but requires both the initial radiation pattern and the damaged radiation pattern and the computational cost is high. Neural networks are effective tools and sometimes can be used as a stage before the genetic algorithm in fault finding. The ANNs bypasses the iterative process when trained properly. The results of the case based reasoning (CBR) system however are very promising besides its capability to reduce the computational cost.

Authors in [28] have proposed a case based reasoning system for fault diagnosis for moderate or large linear antenna arrays and also self organizing map (SOM) to retrieve the cases most similar to the radiation pattern being analysed. This method has been found to be more effective with a success rate of around $93 \%$ as compared to $76.7 \%$ for MLP $76.0 \%$ for RBF. In [29], authors have presented the hybridization of the two approaches; the genetic algorithm (GA) and the back propagation network (BPN) employing a three layer multi layer perceptron (MLP). In this work, the fault finding in antenna arrays has been considered as a mapping and weight optimization problem. A 16-element linear micro strip array having $\square$ inter element spacing of $0.3 \lambda$, simulated at $1.9 \mathrm{GHz}$ has been used in the study. The genetic algorithm determines the weights in a BPN. The genetically trained back propagation network is better in minimizing the error. The hybrid method proves its effectiveness and paves way for combination of techniques.

Authors in [30] have used genetic algorithm based method for 100 dipoles elements with interspacing of $0.5 \lambda$ for detecting complete faults in the arrays. The method has proven its effectiveness for completely faulty as well as partially faulty element. The genetic algorithm is normally used to detect the faulty elements in small size arrays [31] but for large arrays it has to be run several times for accurate results. Applications of radial basis function (RBF) and probabilistic neural network (PNN) have been suggested by authors in [31] and [32] using planar array with $5 \times 5$ and $8 \times 8$ isotropic elements having uniform excitations and inter element spacing of $0.5 \lambda$. The radiation pattern is sampled between angles $-90^{\circ}$ to $90^{\circ}$. The networks are tested by faulty patterns with $3 \%$ and $6 \%$ measurement error. The method proves its effectiveness in fault diagnosis. It is also observed that the efficiency of PNN network is better than RBF. Authors in [33] have proposed a NN based fault finding method using MLP trained with back propagation. The antenna array used is a 6x6 microstrip planar antenna with inter element spacing of $0.9 \lambda$ designed for $16.64 \mathrm{GHz}$ frequency.

\section{Conclusions}

Fault diagnostics in antenna arrays has been quite challenging and tedious for analytical as well as numerical methods specially with analog beamforming approaches. The digital beamforming techniques employed in smart antenna arrays enable array failure correction without needing the replacement of the defective elements. Neural network and genetic algorithms both have been found to be effective in fault diagnosis in antenna arrays. The neural networks perform the fault finding by taking samples of radiation pattern of the array with fault elements and mapping the same to the location of the faulty element in that array. Neural networks operate on simple tools and do not perform tedious processes hence they are found to be faster (after training) in real time applications than the traditional analytical and numerical methods. Neural networks have demonstrated their effectiveness in fault finding applications related with antenna arrays. Genetic algorithms have also been quite effectively employed in fault finding applications for completely and partially faulty elements. Combining the two techniques with their respective features has also been found very effective in fault diagnostics of antenna arrays.

\section{Acknowledgements}

We express our sincere thanks to all the authors and the publishers of the books, research/reviews papers and articles referred in this manuscript.

\section{References}

[1] L. C. Godara, "Smart Antennas", Boca Raton: CRC Press LLC, 2004.

[2] C.A.Balanis, "Antenna Theory, Analysis and Design", $3^{\text {rd }}$ edition, John Wiley and Sons, NJ, 2005.

[3] A. H. El. Zooghby, "Smart Antenna Engineering", Artech House, 2005.

[4] Alexiou and M. Haardt, "Smart Antenna Technologies for Future Wireless Systems: Trends and Challenges", IEEE Communications Magazine, pp. 90-97, Sept 2004. 
[5] L C. Godra, “Application of Antenna Arrays to Mobile Communications Part1: Performance Improvement, Feasibility, and System Considerations", Proceedings of the IEEE vol. 85, no.7 pp. 1031-1060, July 1997.

[6] M.Chryssomallis, "Smart Antennas", IEEE Antennas and Propagation Magazine, vol.42, no.3, pp.129-36,June 2000.

[7] S. Bellofiore, C. A. Balanis, J. Foufz, and A.S. Spanias, "Smart-Antenna Systems for Mobile Communication Networks Part I: Overview and Antenna Design", IEEE Antennas and Propagation Magazine, vol.44, no.3, 2002.

[8] B. Widrow and M.A. Lehr, "A 30 Years of Adaptive Neural Networks: Perceptron, Madaline and Backpropagation", Proceedings of the IEEE, vol.78, issue 9,pp. 1415-42, 1990

[9] Titus K. Y. Lo, Henry Leung and John Litwa, "Artificial Neural Network for AOA Estimation in Multipath Environment Over the Sea", IEEE Journal of Oceanic Engineering, vol. 19, issue 4, pp. 555-562, Oct.1994.

[10] S. Haykins, "Neural Networks: A Comprehensive Foundation", IEEE Press, NY, 1994

[11] H. L. Southhall, J. A. Simmeres, and T. H. O’Donnell, "Direction Finding in Phased Arrays with a Neural Network Beamformer", IEEE Transactions on Antennas and Propagation,vol.43, no.12, Dec.1995.

[12] A. H. El. Zooghby, C. G. Christodoulou, and M. Georgiopoulos, "Performance of Radial Basis Function Networks for Direction of Arrival Estimation with Antenna Arrays”, IEEE Transactions on Antennas and Propagation, vol. 45, no. 11, pp. 1611-1617, Nov. 1997.

[13] M. Ibnkahla, “Applications of Neural Networks to Digital Communications - A Survey" Elsevier Signal Processing Journal, vol. 80 , issue 7, pp.1185-1215, July 2000

[14] Q. J. Zhang and K. C. Gupta, "Neural Networks for RF and Microwave Design", Artech House, 2000.

[15] A. Patnaik, D. E. Anagnostou, R. K. Mishra, C. G. Cristodoulou, and J. C. Lyke, "Applications of Neural Networks in Wireless Communications", IEEE Antennas and Propagation Magazine, vol. 46, no.3, pp. 130-137, June 2004.

[16] M. Sarevska, B. Milovanovic, and Z. Stankovic, "Alternative Signal Detection for Neural Network Based Smart Antenna", Proc. of 7th International Seminar on Neural Network Applications in Elect. Engineering, NEUREL, pp.85-89, 2004

[17] K.L. Du, A. K. Y. Lai , K. K. M. Cheng, and M. N. S. Swamy, "Neural Methods for Antenna Array Signal Processing: A Review", Elsevier Science B. V., Signal Processing, vol. 82, issue 4, pp 547-561, Apr. 2002.

[18] S. Mishra, R. N. Yadav and. R. P. Singh, "Neural Networks in Smart Antenna Design for Co-Channel Interference (CCI) Reduction: A Review", Proceedings of ICWMC 2009, pp. 228-233, Oct.2009.

[19] A.Rawat, R. N. Yadav, and S. C. Shrivastava, "Neural Networks Applications in Smart Antennas Arrays: A Review", International J. of Electronics and Comm. Engineering (AEÜ Elsevier), vol. 66, pp. 903-912, Nov. 2012.

[20] B.K. Yeo and Y. Lu, "Array Failure Correction wtha Genetic Algorithm”, IEEE Transactions on Antennas and Propagation, vol.47, pp.823-828,1999

[21] J. A. Rodriguez, F. Ares, H. Palacios, and J. Vassal'lo, “Finding Defective Elements in Planar Arrays Using Genetic Algorithms", Progress in Electromag. Research, PIER 29, pp. 25-37, 2000.

[22] G. Castaldi, V.Pierro, and I.M. Pinto, "Efficient Faulty Element Diagnostics of Large Antenna Arrays by Discrete Mean Field Neural Nets", Progress in Electromag. Research, PIER 25, pp. 53-76, 2000.

[23] J. A. Rodriguez, F. Ares, and E. Moreno, "Waveguide-fed Longitudinal Slot Array Antennas: Fault Diagnosis Using Measurements of Input Impedance", Microwave and Optical Technology Letters, vol. 32, pp.200-201, 2002.

[24] A.Padron, J.I. Garduno, A.A. Herrara and R. Prieto, "Antenna Array Adjust with Adaptive Neuronal System", Journal of Applied Research and Technology, pp.189-198, 2003.

[25] A. Patnaik, C. Christodoulou, "Finding Failed Element Positions in Linear Antenna Arrays Using Neural Networks", IEEE Antenna Propagation Soc. Symposium, pp. 1675-1678, 2006.

[26] A. Patnaik , B. Choudhury , P. Pradhan , R.K.Mishra , and C.Christodoulou, "An ANN Application for Fault Finding in Antenna Arrays", IEEE Transactions on Antennas and Propagation., vol.55, pp.775-777,2007.

[27] J.A.Rodriguez, M. Fernandez-Delgado, J. Bregains, R. Igesias, S.Barro, and F. Ares, "A Comparison Among Several Techniques for Finding Defective Elements in Antenna Array", EUCAP the second European Conference on Antenna and Propagation, pp. 1$8,2007$.

[28] R. Iglesias R, F. Ares , M.Fernandez-Delgado,J.A. Rodriguez, J. Bregains, and S. Barro, "Element Failure Detection in Linear Antenna Array Using Case Based Reasoning", IEEE Antennas and Propagation Magazine, vol.50, no.4, Aug. 2008.

[29] S. Choudhary, S. Pattanayak, A. Patnaik, "A Genetically Trained Neural Network Application for Fault Finding in Antenna Arrays", Applied Electromagnetics Conference, pp.1-4, 2009.

[30] J. A. Rodriguez, M. Fernandez-Delgado, J. Bregains, R. Igesias, S.Barro, and F. Ares, "Rapid Method for Finding Faulty Elements in Antenna Arrays Using Far Field Pattern Samples", IEEE Transactions on Antennas and Propagation,vol.57,no.6, 2009.

[31] D. Vakula and N. V. S. N. Sarma, "Fault Diagnosis of Planar Antenna Arrays Using Neural Networks", Progress in Electromag. Research M, vol.6, pp.35-46, 2009.

[32] D. Vakula and N. V. S. N. Sarma, "Using Neural Networks for Fault Detection in Planar Antenna Arrays”, Progress in Electromag. Research Letters, vol.14, pp.21-30, 2010.

[33] A. R. Mallahzadehand M. Taherzadeh "Element Failure Diagnosis in a Planar Antenna Array by the use of Neural Networks", pp.294-298, 2010

[34] Y. L. Sit, M. Agatonovic, and T. Zwick, "Neural Network Based Direction of Arrival Estimation for a MIMO OFDM Radar", Proc. of the $9^{\text {th }}$ European Radar Conf., EuRAD, pp.298-301, Oct-Nov 2012.

[35] S. Mishra, R. N. Yadav, and R.P. Singh, "A Survey on Applications of Multi Layer Perceptron Neural Networks in DOA Estimation of Smart Antennas”, International Journal of Computer Applications, vol.83, no.17, pp.22-28, Dec. 2013. 